\title{
Fatores que afetam a intenção em continuar o uso do e-learning: um estudo com professores de uma Universidade Federal
}

Factors affecting intention to continue using e-learning: a study with Federal University teachers

\section{Resumo}

Maria Luíza Azevedo de Carvalho ${ }^{\mathrm{I}}$ Angilberto Sabino de Freitas ${ }^{2}$ Anatália Saraiva Martins Ramos ${ }^{3}$ Thiago Cavalcante Nascimento ${ }^{4}$ Jorge Brantes Ferreira ${ }^{5}$

O estudo objetiva identificar os fatores que influenciam a intenção de docentes de uma instituição de ensino superior (IES) pública federal em continuar o uso do $e$-learning. Por meio de uma extensão do modelo TAM, incluindo tempo e autoeficácia como variáveis externas, foi possível verificar que a intenção em continuar o uso do e-learning é influenciada pela percepção de que a tecnologia pode contribuir para a melhoria do desempenho dos professores. Verificou-se que a intenção em continuar usando o e-learning pode não ocorrer de acordo com os pressupostos indicados pelo modelo TAM.

Palavras-chave: Modelo de Aceitação da Tecnologia, Adoção de Tecnologia, Elearning.

\begin{abstract}
The study aims to identify the factors that influence teachers' intention of continuingto usee-learning in a federal university in Brazil. Through an extension of the TAM model, where was time and self-efficacy were included as external variables, it was found that the intention to continue using e-learning is influenced by the perception that the evaluated technology can contribute to improve teachers' performance. It was also found that the intention to continue to use e-learning methodologies may not occur accordingto some of the constructs used in the TAM model.

Keyword: Technology Acceptance Model, Technology Adoption, E-learning.
\end{abstract}

\footnotetext{
1 malu.adecarvalho@gmail.com, Brasil. Mestranda em Administração de Empresas na Pontifícia Universidade Católica do Rio de Janeiro - PUC-Rio. Rua Marquês de São Vicente, 225, Gávea, CEP: 22451-900 - Rio de Janeiro, RJ - Brasil.

2 angilberto.freitas@gmail.com, Brasil. Professor Adjunto da Universidade do Grande Rio - UNIGRANRIO. Doutor em Administração de Empresas pela Pontifícia Universidade Católica do Rio de Janeiro - PUC-Rio. Rua da Lapa, 86, Lapa, CEP: 20021-180 - Rio de Janeiro, RJ - Brasil

3 anatalia_ufrn@yahoo.com.br, Brasil. Professora Titular da Universidade Federal do Rio Grande do Norte - UFRN. Doutora em Engenharia de Produção pela Universidade Federal do Rio de Janeiro - UFRJ. Campus Universitário, Lagoa Nova, CEP: 59072-970 - Natal, RN - Brasil.

4 thiagocn1@hotmail.com, Brasil. Doutorando em Administração na Universidade Federal do Paraná - UFPR. Av Lothário Meissner, 632, Jardim Botânico, CEP: 80210-170 - Curitiba, PR - Brasil.

5 jorge.brantes@gmail.com, Brasil. Professor Assistente da Pontifícia Universidade Católica do Rio de Janeiro - PUC-Rio. Doutor em Administração de Empresas pela Universidade Federal do Rio de Janeiro - UFRJ. Rua Marquês de São Vicente, 225, Gávea, CEP: 22451-900 - Rio de Janeiro, RJ - Brasil.
}

Recebido em 16.10.2012

Aprovado em 27.03.2013

Revista Administração em Diálogo

ISSN 2178-0080

Programa de Estudos Pós-Graduados em Administração

Pontifícia Universidade Católica de São Paulo 


\section{Introdução}

Com o desenvolvimento das novas Tecnologias de Informação e Comunicação (TIC's) e o avanço da rede mundial de computadores a partir de I992 (HARASIM, 2OOO), as duas últimas décadas experimentaram uma enorme expansão do uso de tecnologia no ambiente de ensino, ou e-learning, como é comumente chamado (NICHOLS, 2008), podendo ser usadas tanto em modelos de educação a distância (EAD) quanto para melhorar o processo de ensino-aprendizagem no ambiente presencial (FREITAS, 2009).

Entretanto, o mero fato de introduzir a tecnologia para fins de ensino e aprendizagem não garante que ela será adotada pelos usuários, o que pode levar a uma dificuldade de sua difusão dentro das organizações (FREITAS, 2009). Nesse contexto, compreender os fatores que influenciam como os docentes avaliam o uso de tecnologias no ambiente de ensino é crucial para prepará-los para esta nova realidade. Assim, é postulada a seguinte questão de pesquisa: Que fatores podem influenciar os docentes a continuar o uso do e-learning? Para responder a essa questão, são propostos dois objetivos: (I) identificar um modelo adequado para avaliar a aceitação de tecnologia por usuários e (2) por meio do modelo identificado, avaliar a intenção em continuar o uso do e-learning por docentes em uma IES que introduziu a tecnologia para a EAD.

O artigo está estruturado da seguinte forma: inicia-se com esta introdução, que contextualiza o problema de pesquisa e expõe os objetivos do trabalho. Em seguida, conceitua-se o que é o e-learning, para então revisar modelos de adoção de tecnologia existentes na literatura, propondo um modelo para avaliar a intenção de uso do $e$ learning em uma IES no Brasil. Na seção seguinte é descrita a metodologia, seguida da análise e discussão dos resultados. O trabalho encerra-se com as considerações finais do artigo.

\section{Revisão da Literatura}

\section{O E-learning}

Por ser um fenômeno recente, diferentes áreas possuem entendimentos particulares sobre o significado do e-learning, dificultando definir exatamente o que ele significa. A sua implementação em diversas áreas, a multiplicidade de tecnologias e as 
diferentes formas de uso tornam a definição do termo ainda mais difícil. Facilmente confunde-se com uma série de outras expressões, como "treinamento online", “treinamento baseado na Web" e "treinamento baseado no computador". O problema é que essa indefinição pode causar confusão no entendimento do que significa o $e$-learning, quais são os seus objetivos, qual a sua relação com a tecnologia e com a educação (ZEMSKY; MASSY, 2004). É muito comum os termos "educação a distância”, "ensino a distância", "ensino mediado por computador", "ensino online”, "ensino distribuído", “ensino baseado na web” serem usados de forma intercambiável, quando na verdade muitos deles representam coisas distintas (GURI-ROSENBLIT, 2005). Pode-se fazer EAD usando e-learning como ferramenta, como se pode fazer EAD sem e-learning, bem como usar $e$-learning e não necessariamente adotar um modelo de EAD.

Para Gilbert e Jones (2OOI), e-learning é a distribuição de conteúdo educacional via qualquer meio eletrônico, incluindo internet, intranets, extranets, transmissão via satélite, gravações de áudio e vídeo, TV interativa, CD-ROM e treinamento mediado por computador. De maneira geral, o conteúdo é disponibilizado de forma online, onde os indivíduos podem navegar no ambiente e o aprendizado ocorre via internet. Pode-se perceber que nessa definição a ênfase está mais no aspecto de distribuição do material do que propriamente na questão da pedagogia em si. Seguem essa corrente de que o $e$ learning é uma ferramenta que facilita a distribuição Henry (2OOI), Rosenberg (2008) e Siritongthaworn et al. (2006).

Já na visão de Guri-Rosenblit (2005), o e-learning é um fenômeno relativamente novo no qual se relaciona com o uso das TIC’s para uma variedade de propósitos de uso que vão desde a simples distribuição de conteúdo online até a substituição completa de encontros presenciais por aulas totalmente virtuais. Para ela, toda e qualquer forma de ensino e aprendizado por meio das TIC's é referenciada como e-learning. Contudo, Guri-Rosenblit deixa claro que a relação do uso da tecnologia no processo de ensino deve enfatizar a importância do e-learning estar ligado à questão pedagógica. Entretanto, não faz distinção a que modelos pedagógicos seriam mais adequados ao uso do $e$-learning.

Nessa mesma linha, Nichols (2008) define e-learning como pedagogia alavancada por meio do uso de tecnologia digital. Segundo ele, e-learning é uma combinação de e-(de eletrônico) e aprendizagem, mas sempre dirigido pela pedagogia. A 
tecnologia (entendida como tecnologia digital) às vezes permite novas pedagogias, mas esta deve ser sempre definida antes para lhe dar direcionamento. Para o e-learning ocorrer, pedagogia eficiente deve ser combinada com tecnologia de fácil utilização. Assim, o e-learning é fortemente dependente de pedagogia. Se esta é ineficiente, ou há pouca ou nenhuma preocupação com a pedagogia, o uso da tecnologia no processo de ensino será ineficaz (NICHOLS, 2008).

À medida que computadores e equipamentos eletrônicos invadem os ambientes de aprendizagem, oferecendo inovações de imagem, som, movimento, hipertextualidade, virtualidade e realidade virtual, faz-se necessário repensar as formas de interação entre professores e alunos (FILATRO, 2003). O surgimento da aprendizagem online e de ambientes baseados na web tem contribuído significativamente para o reconhecimento da necessidade de uma maior compreensão das condições de como a tecnologia é apropriada por docentes e discentes, e como ela pode ser usada para atingir uma aprendizagem mais eficaz.

Entretanto, é importante notar que nos contextos de e-learning é exigido que novas competências sejam aprendidas (HARASSIM et al., 2005), o que pode levar à resistência por parte dos usuários em adotá-la, dificultando o uso da tecnologia para fins de ensino e aprendizagem. Dessa forma, pode-se supor que a difusão do e-learning nas organizações de ensino pode depender ou não dos usuários estarem dispostos a adotarem a nova tecnologia que é diferente do que eles estão acostumados no processo tradicional de ensino presencial. Esse aspecto remete a compreender como a introdução de tecnologias de informação e comunicação pode ser aceita pelos indivíduos. Nesse sentido, diversos estudos buscam compreender que fatores influenciam como o usuário pode aceitar (ou não) essas tecnologias. Esse arcabouço teórico para avaliar a aceitação de tecnologia é apresentado a seguir.

\section{A Aceitação de Tecnologia}

Historicamente, o foco principal das diversas linhas de pesquisa sobre aceitação de tecnologia esteve na implementação e utilização de novas tecnologias de informação no ambiente de trabalho e para tarefas produtivas, buscando analisar os fatores que influenciavam a utilização ou a intenção de seu uso (AGARWAL; PRASAD, I999; 2OOO; 
DAVIS et al., I989; IGBARIA et al., I995; MATHIESON, I99I; VENKATESH, I999; VENKATESH; DAVIS, 200o; VENKATESH et al., 2003). Entretanto, a utilização desses modelos tem sido estendida com sucesso para outros contextos fora do ambiente organizacional, como os trabalhos de Childers et al. (2OOI), avaliando o uso de internet por consumidores e de Suh e Han (2003) sobre o uso de comunidades virtuais.

A base teórica para esses modelos se desenvolveu a partir da Teoria da Ação Intencional (do inglês Theory of Reasoned Action - TRA) de Fishbein e Ajzen (I975), da qual derivaram, entre outros, a Teoria do Comportamento Planejado (Theory of Planned Behaviour - TPB) de Ajzen (I99I), o Modelo de Aceitação de Tecnologia (Technology Acceptance Model - TAM) de Davis (I989) e Davis et al. (I989) e a Teoria Unificada de Aceitação e Uso de Tecnologia (Unified Theory of Acceptance and Use of Technology UTAUT) de Venkatesh et al. (2003), que é uma tentativa de unificar diversos modelos anteriores.

\section{Teorias que deram origem aos modelos de aceitação de tecnologia}

A Teoria da Ação Intencional (TRA) de Fishbein e Ajzen (I975) (figura I) é um modelo originário da psicologia social e foi desenvolvida para explicar o comportamento humano, não sendo um modelo proposto exclusivamente para avaliar o processo de aceitação de tecnologias computacionais. O modelo estabelece que o desempenho de um determinado comportamento de um indivíduo é determinado por suas intenções comportamentais e esta intenção comportamental, por sua vez, é determinada por dois aspectos: a sua atitude e as normas subjetivas em que operam.

Figura I: Teoria da Ação Intencional.

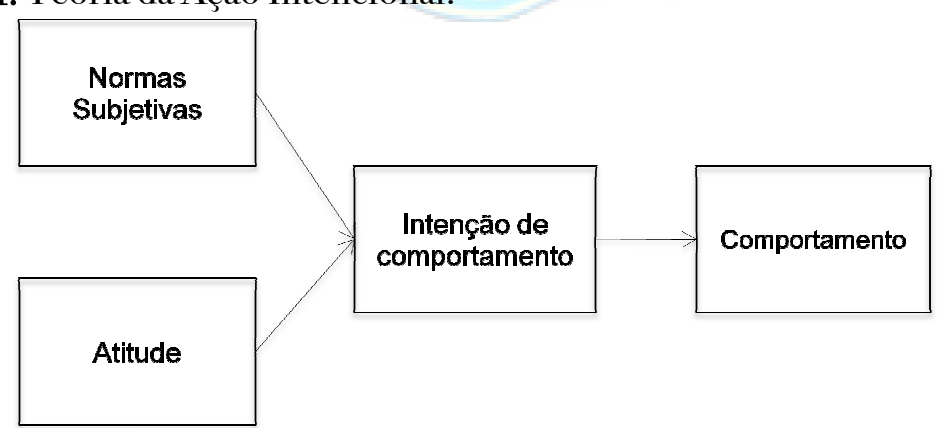

Fonte: Adaptado de Fishbein\& Ajzen (1975). 
Segundo Fishbein e Ajzen (1975), a atitude pode ser definida como os sentimentos, positivos ou negativos que um indivíduo possui em relação a um comportamento alvo, e a intenção de comportamento reflete a sua disposição pessoal em se engajar em um comportamento, representando sua avaliação pessoal de acordo com suas crenças que, favorável ou desfavoravelmente, influenciam essa intenção. O outro componente, normas subjetivas, se refere à percepção que um indivíduo tem sobre a opinião de pessoas que lhe são importantes a respeito do fato de que ele deva ou não adotar o comportamento em questão, isto é, está calcado na percepção de uma avaliação a respeito de adotar ou não determinado comportamento (FISHBEIN; AJZEN, I975).

Ajzen (I99I) estende o modelo TRA com o objetivo de fazer uma predição da intenção do comportamento e do comportamento em situações no qual um indivíduo possui controle incompleto. Assim, um terceiro construto é introduzido independente da atitude a das normas subjetivas: o controle comportamental percebido, e o modelo resultante foi chamado de Teoria do Comportamento Planejado (TPB) (figura 2). O controle comportamental percebido reflete a crença que um indivíduo detém sobre a disponibilidade de recursos e de oportunidades (ou fatores que dificultam ainda mais o desempenho do comportamento). Em conjunto, a atitude, as normas subjetivas e percepção de controle comportamental contribuem diferentemente para a formação da intenção comportamental que se presume ser o antecedente do comportamento.

Figura 2: Teoria do Comportamento Planejado (TPB)

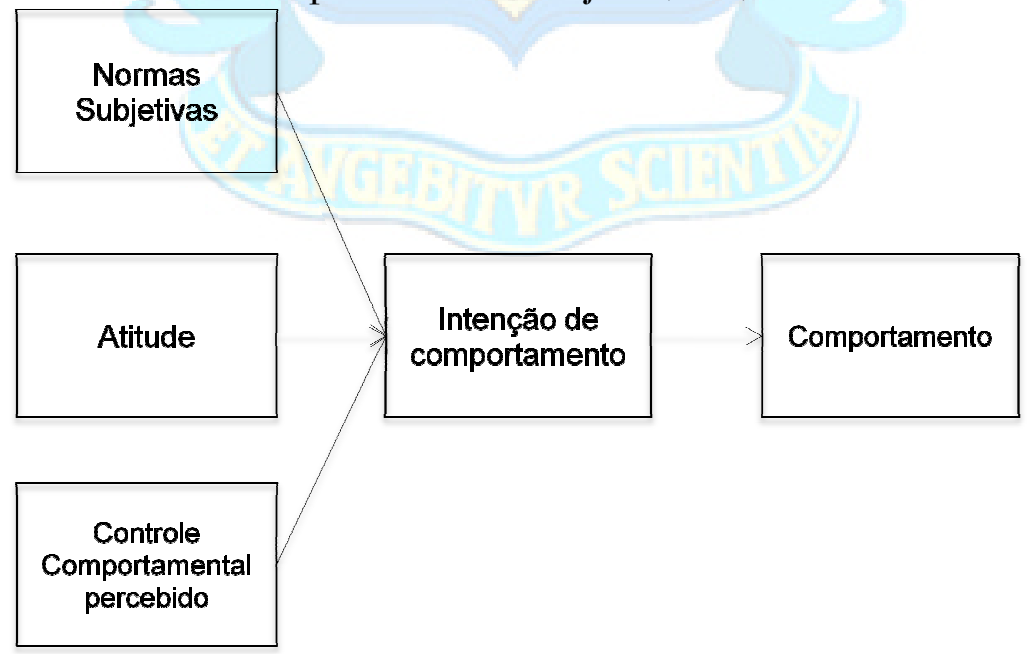

Fonte: (Ajzen, I99I). 
Com base nesses modelos, foram desenvolvidos diversos outros para avaliar particularmente o uso de tecnologias associadas a sistemas de informação e comunicação. Dois modelos tem se destacado nessa literatura: o Modelo de Aceitação de Tecnologia (TAM) de Davis (I989) e Davis et al. (I989) e a Teoria Unificada de Aceitação e Uso de Tecnologia (UTAUT) de Venkatesh et al. (2003).

\section{O modelo de aceitação de tecnologia (TAM)}

O Modelo de Aceitação de Tecnologia (TAM) (Davis, I989), derivado da Teoria da Ação Intencional, foi concebido como um modelo parcimonioso, desenvolvido especificamente para avaliar a adoção de tecnologia de computadores e tecnologias relacionadas. O seu argumento teórico enfatiza duas variáveis que influenciam a atitude: a utilidade percebida e a percepção de facilidade de uso. Dentro do modelo, a utilidade percebida significa medida pela qual as pessoas acreditam que a tecnologia vai ajudá-las a fazer um trabalho melhor. Já a utilidade percebida é caracterizada como a probabilidade de melhoria de desempenho em tarefas relacionadas ao seu trabalho que o indivíduo enxerga ser possível por meio do uso de uma dada tecnologia. Posteriormente, essa definição foi ampliada para contextos fora do ambiente de trabalho, com a utilidade passando a significar simplesmente as melhorias percebidas por um indivíduo em sua produtividade ou eficiência em qualquer tarefa, proporcionadas pelo da tecnologia. Já a percepção de facilidade de uso representa a percepção que o indivíduo possui acerca do esforço que terá que despender para usar a nova tecnologia. Essas duas variáveis espelham duas das características da inovação descritas no modelo de difusão de Rogers: vantagem relativa e complexidade. Posteriormente, Davis et al. (I989) expandiram o modelo, chamado de TAM 2 ou TAM estendido, sugerindo a inclusão de variáveis externas a ele (Figura 3 ). 
Figura 3: TAM2 - Modelo de Aceitação de Tecnologia

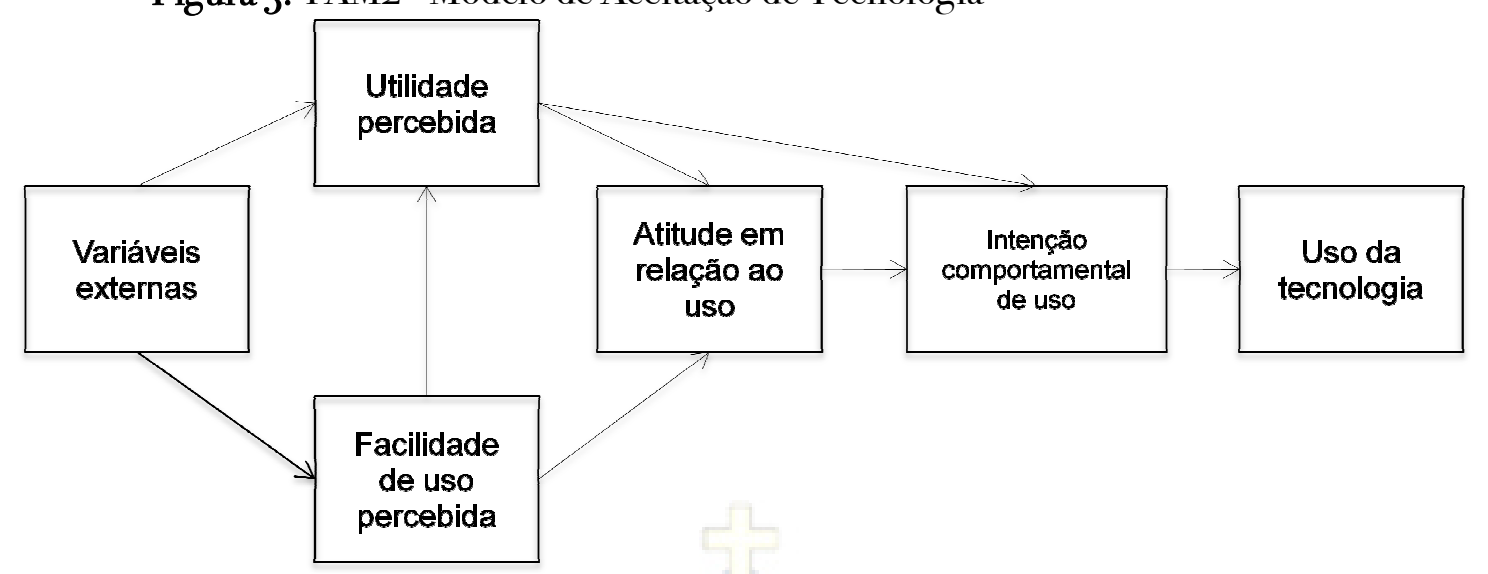

Fonte: (Davis et at, I989).

No entanto, os próprios autores do TAM original (DAVIS et al., I989), ao testar o modelo sem o construto atitude, em ambientes onde a escolha do uso da tecnologia era mandatória (por exemplo, em empresas que adotam um novo sistema que deve ser obrigatoriamente usado por todos os funcionários), perceberam que o modelo se comportava igualmente bem com ou sem a atitude mediando a intenção de uso. Em cenários onde o uso da tecnologia é voluntário, a atitude se mostrou relevante e um fator determinante do uso, apesar de sua relação direta com a intenção de uso não ficar comprovada (JACKSON et al., I997). Uma possível explicação para a conclusão de que a atitude não é um construto essencial para o TAM, de acordo com Taylor e Todd (1995b), é o fato de que, para ambientes de trabalho, o desempenho da tecnologia é o fator mais importante, com os gostos ou sentimentos pessoais dos possíveis usuários não exercendo influência significativa sobre a intenção de uso ou o uso concretizado.

Testado exaustivamente ao longo dos anos no campo de sistema de informações e tendo servido de base para a criação de vários modelos posteriores (KULVIWAT et al., 20O7), Yousafzaietal. (20O7) atestam que a grande popularidade do TAM pode estar associada ao fato do modelo ser parcimonioso, específico para o contexto de inovações tecnológicas e capaz de produzir explicações e predições adequadas para a aceitação de diversas tecnologias em diferentes ambientes. Além disso, o TAM possui uma base sólida teórica e várias escalas validadas para a medição de seus construtos, facilitando a sua aplicação e replicação nas mais distintas condições e contextos. 


\section{Teoria Unificada de Aceitação e Uso de Tecnologia (UTAUT)}

Em uma tentativa de propor um modelo de aceitação, uso e difusão de tecnologia mais abrangente, Venkatesh et al. (2003) propõem o modelo UTAUT - Teoria Unificada de Aceitação e Uso de Tecnologia -, no qual integra elementos de oito teorias diversas: (I) a Teoria da Ação Intencional (TRA) (FISHBEIN;AJZEN, I975);(2) o Modelo de Aceitação de Tecnologia (TAM) (DAVIS et al., I989) (3) o Modelo Motivacional (MM) (DAVIS et al., I992); (4) a Teoria do Comportamento Planejado (TPB) (AJZEN, I99I); (5) o TAM combinado com o TPB (C-TAM-TPB) (TAYLOR; TODD, I995a); (6) o Modelo de Utilização de PG (MPCU) (TRIANDIS, I977; THOMPSON, et al., I99I); (7) a teoria da difusão da inovação (ROGERS, 2003; MOORE;BENBASAT, I99I); e (7) a teoria cognitiva social (BANDURA, I986; COMPEAU; HIGGINS, I995).

Venkatesh et al. (2003) teorizam que quatro construtos: (I) expectativa de desempenho, (2) expectativa de esforço, (3) influência social e (4) condições facilitadoras, todos moderados pela idade, experiência, sexo e voluntariedade de uso, teriam influência direta sobre a intenção de uso e o uso efetivo da tecnologia. O modelo (Figura 4) foi elaborado e testado para modelar a adoção de novos sistemas e tecnologias da informação em ambientes de trabalho, na mesma linha do TAM original.

Figura 4: Teoria Unificada de Aceitação e Uso de Tecnologia

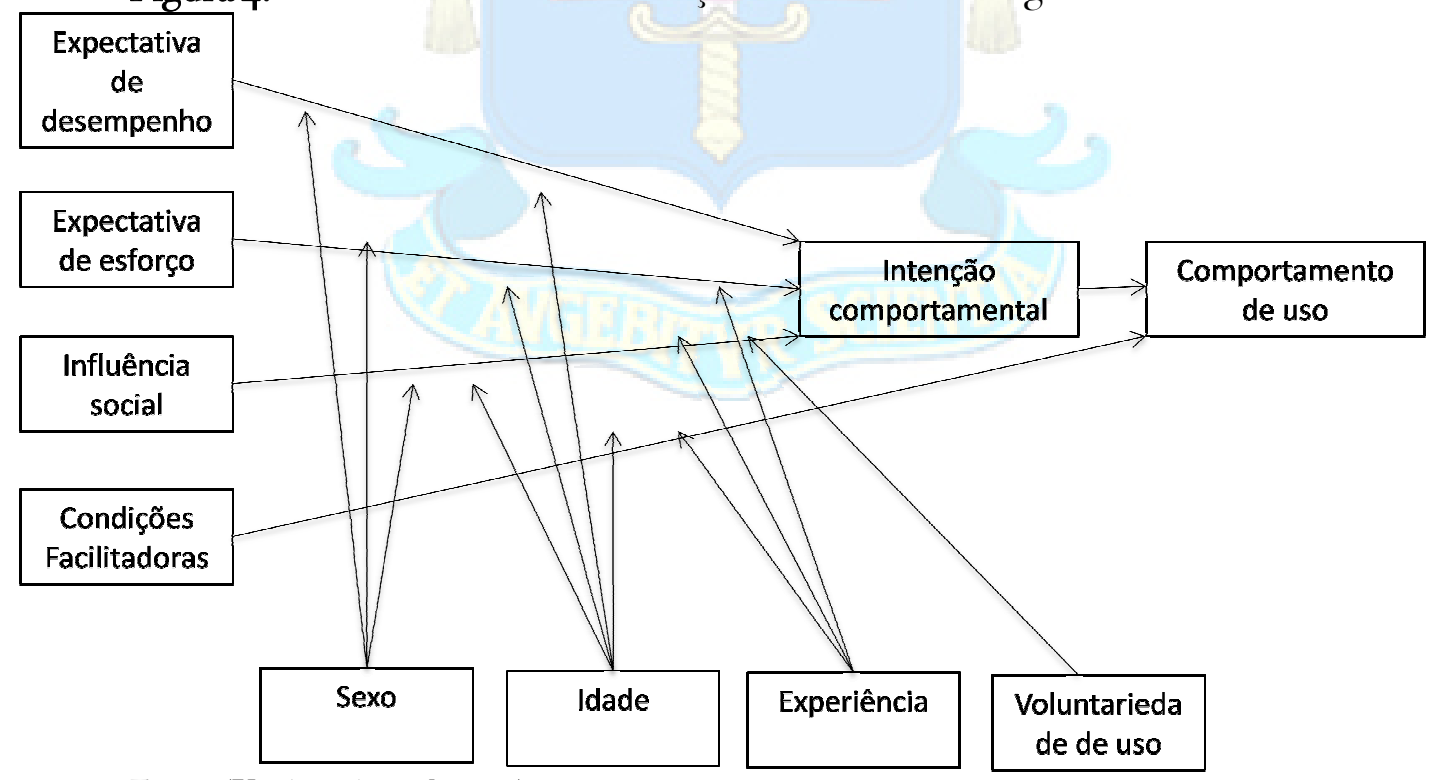

Fonte: (Venkatesh etal, 2003). 
Segundo Venkatesh et al. (2003), a expectativa de desempenho é definida como o grau em que um indivíduo acredita que usar uma tecnologia irá ajudá-lo a alcançar níveis superiores de desempenho em suas tarefas de trabalho. É baseado no construto utilidade percebida do TAM (DAVIS et al., I989) e no construto vantagem relativa da teoria de difusão de inovações de Rogers (2003).

Já a expectativa de esforço é definida como o grau de facilidade associado ao uso de uma tecnologia e é similar a construtos presentes em outros modelos, como a facilidade de uso percebida ou o construto complexidade, também presente na teoria de difusão de inovações de Rogers. Venkatesh et al. (2003) afirmam que o construto expectativa de esforço (e seus similares em outros modelos) é significante na previsão da intenção de uso, particularmente nos primeiros estágios da adoção (THOMPSON et al., I994).

Por sua vez, o construto influência social representa o grau de percepção de um indivíduo sobre o quão importante é para pessoas próximas e relevantes que ele utilize a tecnologia. É similar ao construto normas subjetivas presente na TRA de Fishbein e Ajzen (1975) e na TPB de Ajzen (I99I).

Por fim, o construto condições facilitadoras mede até que ponto um indivíduo acredita que a infraestrutura organizacional e técnica existente na empresa permita e suporta a utilização da tecnologia a ser adotada. Esse construto é similar ao construto controle percebido da TPB (AJZEN, I99I) e ao construto compatibilidade da teoria de difusão de inovações de Rogers (2003).

Venkatesh et al. (2003) testaram o UTAUT empiricamente e os resultados apresentaram forte suporte para as relações e construtos propostos no modelo, com efeitos diretos significativos sobre a intenção de uso da tecnologia sendo verificados para os seguintes construtos: expectativa de desempenho, expectativa de esforço e influência social. Além disso, foram confirmados efeitos diretos da intenção de uso e do construto condições facilitadoras sobre o uso concretizado da tecnologia. Assim, o UTAUT apresenta uma sólida base teórica e sugere construtos que poderiam ser utilizados e testados também para a adoção de tecnologias de comunicação e informação em outros contextos.

O arcabouço teórico apresentado acima descreve a origem de dois dos principais 
modelos encontrados na literatura para analisar o comportamento e a intenção de uso de tecnologia informação em geral, sendo, principalmente o TAM, amplamente adotado para verificar o uso dessas tecnologia nos ambientes de ensino, e por isso será adaptado para atingir os objetivos desta pesquisa.

Aplicações dos modelos de aceitação de tecnologia ao ambiente de ensino

Por considerar que as tecnologias de informação e comunicação usadas para o processo de ensino e aprendizagem são, de fato, tecnologias da computação ou relacionadas, diversos pesquisadores tem usado o arcabouço teórico exposto acima para investigar o processo de aceitação e difusão das tecnologias digitais no ambiente de ensino.

No que diz respeito ao e-learning, o uso desses modelos tem sido amplamente documento na literatura estrangeira (ARBAUGH, 2005; CHENG et al., 2OII; HONG et al., 2OII; HUANG et al., 2OI2; KIRAZ; OZDEMIR, 2006; MARTINS; KELLERMANNS, 2004; PITUCH; LEE, 2006; SANCHEZ-FRANCO, 20IO; SUGAR et al., 2005; TEO; NOYES, 2OII).

Entretanto, no Brasil ainda é incipiente a pesquisa e os resultados são inconclusivos, principalmente pelo fato do e-learning ainda ser um fenômeno relativamente recente dentro das organizações de ensino. Vasconcelos (2008) analisou a implementação do e-learning em uma organização corporativa utilizando o modelo TAM proposto por Davis et al. (I989). Em seu modelo, incluiu o constructo Ambiente Propício à Aprendizagem como variável externa. Ramos e Oliveira (2OIO) pesquisaram uma amostra de alunos de graduação na modalidade a distância para identificar se a variável gênero influencia a aceitação e o uso de um ambiente virtual de aprendizagem através dos construtos do TAM, facilidade de uso e utilidade percebida e autoeficácia.

Já Brauer (2008) analisou as principais dimensões de resistência ao e-learning na educação corporativa utilizando o modelo UTAUT, concluindo que as dimensões Autoeficácia e Expectativa de Desempenho influenciam direta e positivamente a resistência ao e-learning na educação corporativa, e as dimensões Expectativa de Esforço, Condições Facilitadoras e Interatividade são construtos antecedentes à Expectativa de Desempenho. Utilizando também o UTAUT, Ramos e Oliveira (2OI2) estudaram os 
fatores que afetam servidores públicos a cursarem uma capacitação a distância.Dentre os resultados do estudo foi visto que a crença que o servidor possui no uso do ambiente virtual de aprendizagem (AVA) como forma de melhorar o desempenho de seu trabalho (expectativa de desempenho) foi o fator para sua intenção de uso e de seu uso efetivo. Foi visto também que, no âmbito de uso voluntário da tecnologia, o círculo social do aluno (influência social) não possui efeito em sua intenção em usar o AVA. Além disso, os fatores relacionados com a expectativa de esforço e as condições facilitadoras não tiveram impacto sobre a intenção do uso e de uso efetivo do AVA pesquisado.

\section{Um modelo para avaliação do e-learning}

Com base na literatura apresentada, o modelo TAM foi adaptado neste estudo para avaliar a intenção em continuar o uso do e-learning para fins de EAD por parte dos docentes dentro do ambiente de uma IES pública. É sugerida a introdução de dois construtos externos: restrição temporal (AMERICA, 2006) e autoeficácia (COMPEAU; HIGGINS, I995; PITUCH; LEE; 2006). A variável que considera o tempo como restrição representa um importante elemento visto que, para que uma inovação tecnológica possa ser difundida em ambientes de ensino, é necessário identificar a percepção que se tem à respeito do tempo que se dispenderá para seu aprendizado e uso. Assim, o tempo percebido como necessário para aprender e se tornar proficientes em novas competências é susceptível de afetar a forma como os docentes podem perceber a utilidade do sistema e a sua facilidade de uso (AMERICA, 2006). A partir dessa visão, hipotetiza-se que o tempo afeta diretamente a percepção de facilidade de uso (HI) e a percepção de utilidade $\left(\mathrm{H}_{2}\right)$, pois a restrição temporal parao usuário aprender a usar o sistema pode afetar a sua intenção de uso.

Por sua vez, a autoeficácia (COMPEAU; HIGGINS, I995; PITUCH; LEE; 2006) representa o julgamento de uma pessoa sobre sua própria capacidade de organizar e executar um curso de ação necessário para atingir tipos designados de desempenho. Ainda, conforme descreve Bandura (I977), a autoeficácia reflete a crença sobre a capacidade do indivíduo em executar certas tarefas com sucesso. Argumenta-se que o $e$ learning exige maior autonomia do indivíduo tanto de discentes quanto de docentes (PITUCH; LEE, 20o6). Assim, já tendo sido previamente testada e validada para o $e$ - 
learning (PITUCH; LEE, 20o6), é hipotetizado, que a autoeficácia é um construto importante e influencia positivamente na intenção de uso, mas de forma indireta. Diretamente afetaria a facilidade de uso percebida $\left(\mathrm{H}_{3}\right)$ e a percepção de utilidade de uso $\left(\mathrm{H}_{4}\right)$ e, consequentemente, indiretamente a intenção de uso.

Esses dois construtos foram utilizados na pesquisa pelo fato da amostra ser compreendida por professores que ainda atuam em sua maioria no ensino tradicional (presencial), portanto, viu-se a necessidade de identificar se o tempo seria importante na prática da docência a distância - já que a mesma atua como uma atividade "extra" na carga horária da maior parte dos professores participantes dessa pesquisa, e também de compreender se os professores julgam-se aptos a exercerem essa atividade.

As outras hipóteses são derivadas diretas do modelo TAM, que propõe que a facilidade de uso percebida influencia positivamente a intenção em continuar o uso do $e$ learning $\left(\mathrm{H}_{5}\right)$ e a utilidade de uso percebida influencia positivamente a intenção em continuar o uso do e-learning (H6) (DAVIS et al., I989). Por outro lado, Davis et al. (I989) identificaram que a atitude não é relevante em ambientes em que o uso da tecnologia é mandatório, como nesse caso em que foi instituída a obrigatoriedade de se usar a tecnologia para a EAD. Portanto, o construto atitude não é previsto no modelo. Assim de, acordo com a revisão da literatura, acredita-se que o modelo proposto (figura 5 ) seja adequado para avaliar o e-learning de acordo com as condições locais. O quadro I apresenta o resumo das hipóteses:

Figura 5: Modelo para avaliação do e-learning para o contexto brasileiro.

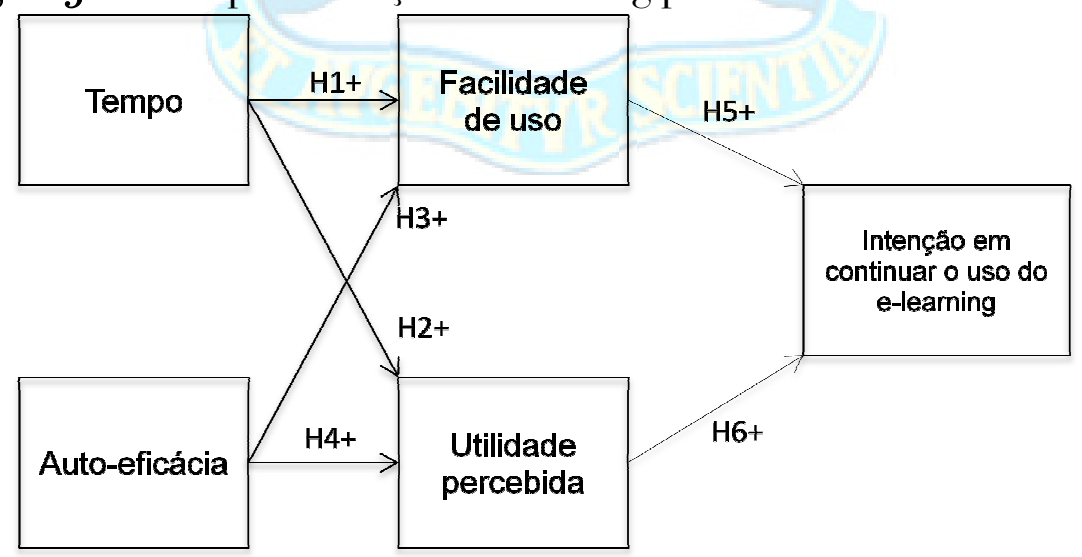

Fonte: Adaptado de Davis et al. r989. 
Quadro I - Resumo das Hipóteses do Estudo

\begin{tabular}{|l|l|}
\hline Hipóteses & \multicolumn{1}{|c|}{ Descrição } \\
\hline H1 & O tempo influencia positivamente a percepção de facilidade de uso. \\
\hline H2 & O tempo influencia positivamente a percepção de utilidade de uso. \\
\hline H3 & A autoeficácia computacional influencia positivamente percepção de facilidade de uso. \\
\hline H4 & A autoeficácia computacional influencia positivamente a percepção de utilidade de uso. \\
\hline H5 & A facilidade de uso percebida influencia positivamente a intenção em continuar o uso do e-learning. \\
\hline H6 & A utilidade de uso percebida influencia positivamente a intenção em continuar o uso do e-learning. \\
\hline
\end{tabular}

Fonte: Elaboração dos autores

\section{Procedimentos Metodológicos}

A presente pesquisa busca identificar quais os fatores que afetam a intenção dos professores em continuar o uso do e-learning por força do curso de graduação a distância de uma universidade federal. Para a consecução do objetivo proposto adotou-se uma abordagem quantitativa de análise, uma vez que se trabalhou essencialmente com escalas numéricas e, caráter descritivo-exploratório, em que variáveis foram observadas, registradas e analisadas no decorrer do estudo (MALHOTRA, 2006).

A população-alvo da pesquisa foi formada por professores de todos os cursos na modalidade à distância oferecidos pela instituição que lecionaram no segundo semestre de 2009 nos cursos de Química, Física, Geografia, Biologia, Matemática e Administração, representando um universo de 84 indivíduos. Desse universo, 76 professores participaram da pesquisa, respondendo ao instrumento de coleta de dados. A aplicação dos questionários ocorreu in loco e se deu de acordo com o critério de conveniência. O total de participantes representou mais de $90 \%$ dos professores e correspondeu a um erro amostral inferior a 2,5\% levando-se em consideração 95\% de confiança para os procedimentos de amostragem finita.

O instrumento de coleta de dados foi constituído por escalas ordinais e nominais segmentadas em dois blocos: o primeiro foi formado por dimensões relacionadas à adoção de tecnologia, como facilidade de uso, utilidade de uso, autoeficácia computacional e tempo de uso, totalizando iz itens, com as escalas dos construtos (o Quadro 2 demonstra a referência de cada construto); a segunda parte estava relacionada 
à caracterização do perfil sócio-demográfico dos professores e suas características em relação ao uso do sistema,

Os itens elencados no instrumento de coleta de dados foram mensurados por meio de uma escala do tipo Likert de sete pontos, variando de discordo totalmente a concordo totalmente e os dados coletados foram tabulados e analisados pelo software SPSS $\AA$.

O processo de tratamento e análise dos dados ocorreu por meio dos procedimentos de estatística descritiva, como média e desvio padrão para possibilitar uma visão geral dos dados, e inferencial com o intuito de verificar as hipóteses norteadoras do estudo; nesta etapa, foram empregadas as técnicas de análise fatorial e regressão linear.

A técnica de análise fatorial foi utilizada para confirmar a adequação das escalas e para a criação de variáveis latentes que explicassem o agrupamento dos indicadores presentes em cada um dos construtos teóricos da pesquisa (HAIR et al., 2005). Para a verificação da consistência interna das variáveis que compunham cada um dos fatores utilizou-se o de Alpha de Cronbach.

Por sua vez, os procedimentos de regressão linear foram utilizados para verificar a existência de relações entre as variáveis estudadas, segundo as hipóteses norteadoras do estudo (MALHOTRA, 2006; HAIR et al., 2005).

\section{Análise dos Dados e Discussão dos Resultados}

Inicialmente, os resultados revelaram que pouco mais de $55 \%$ dos professores são do gênero masculino e que cerca de $37 \%$ deles possuem entre 35 e 45 anos de idade. Dos professores respondentes, mais de $5^{2 \%}$ têm entre seis meses e cinco anos de trabalho na instituição. Cabe salientar que mais de $7_{2} \%$ desses professores possuem doutorado concluído.

Os dados mostraram que cerca de $40 \%$ ministraram apenas uma disciplina nos cursos de EAD da instituição até o momento e outros 30\% com duas disciplinas ministradas. Isto ocorre como uma consequência de que a grande maioria dos professores são convidados pelas coordenações dos cursos de forma esporádica para lecionar uma disciplina específica. 
No que concerne ao uso de tecnologias da informação utilizadas nas tarefas relacionadas às disciplinas, como editores de textos, planilhas, entre outros, os professores acreditam serem muito ativos ou ativos, totalizando mais de $82 \%$ das opiniões. Em oposição a esta informação, verificou-se que, das ferramentas disponibilizadas na plataforma Moodle, que vem a ser o sistema tecnológico onde ocorre o ensino online (MOODLE, 2009), o fórum, o sistema de carregamento de arquivos e as tarefas são as ferramentas mais utilizadas, deixando as demais opções subutilizadas, isto é, apesar dos professores se julgarem muito ativos, deixam de utilizar ferramentas mais avançadas que juntas poderiam minimizar as dificuldades da aprendizagem mediada pelo computador.

Por meio da análise média dos indicadores relacionados às hipóteses do estudo verificou-se uma elevada intenção em continuar utilizando o e-learning como uma ferramenta em suas disciplinas. Três pontos se destacam: (I) pelo padrão de respostas, os professores acreditam que se sentiriam mais a vontade em trabalhar com a tecnologia para a EAD se tivessem passado por um treinamento anterior que lhes ensinassem como proceder em relação às especificidades dessa modalidade de ensino e da tecnologia em si; (2) a existência de certa descrença de que os métodos de EAD sejam mais eficazes do que o modelo tradicional de aprendizagem presencial e; (3) a elevada concordância com a afirmação relacionada ao fato de que os professores poderiam tentar incluir recursos mais sofisticados disponibilizados na plataforma caso observassem outras pessoas as utilizando.

Com base em uma análise fatorial exploratória, foi realizada uma análise das comunalidades obtidas por cada uma das variáveis, bem como das medidas de adequabilidade da amostra (MSA). Por meio deste último teste, verificou-se um problema em um dos itens que compunha a dimensão "Autoeficácia”, o que resultou em sua exclusão e, consequentemente, a criação de uma variável latente que agrupou apenas dois itens.

As premissas da análise fatorial exploratória foram satisfeitas (MALHOTRA, 2006; HAIR et al., 2005), com um KMO igual ou superior a o,5 e um teste de esfericidade de Bartlett significativo (p-valor < o,ooI). Não se utilizou nenhum método específico de rotação, ortogonal ou oblíqua. 
A maioria das cargas fatoriais obtidas foram superiores a o,6 (Quadro 2), demonstrando elevada correlação das variáveis originais com os fatores criados para verificação das hipóteses do estudo. Contudo, em um dos casos obteve-se uma carga negativa, indicando, assim, contrariedade em relação às demais do fator. A partir deste procedimento foram criadas as variáveis métricas utilizadas ao longo dos processos de regressão linear para verificação das hipóteses apresentadas na figura 5 .

Quadro o2 - Síntese da Análise Fatorial

\begin{tabular}{|c|c|c|c|}
\hline Fatores/Referências & Indicadores & $\begin{array}{c}\text { Carga } \\
\text { Fatorial }\end{array}$ & KMO \\
\hline \multirow{3}{*}{ TEMPO (America, 2006) } & $\begin{array}{l}\text { Ensinar através de EaD exige mais habilidade em gerenciar meu } \\
\text { tempo. }\end{array}$ & 0,746 & \multirow{3}{*}{0,625} \\
\hline & $\begin{array}{l}\text { Trabalhar com EaD necessita que eu aumente o tempo que dedico à } \\
\text { realização de atividades de ensino. }\end{array}$ & 0,695 & \\
\hline & $\begin{array}{l}\text { Se eu tivesse mais tempo, poderia me dedicar em aprender a utilizar } \\
\text { mais recursos que o EaD oferece. }\end{array}$ & 0,776 & \\
\hline \multirow{2}{*}{$\begin{array}{c}\text { AUTOEFICÁCIA (Yuen } \\
\text { e Ma, 2008; Gong, Xu e } \\
\text { Yu, 2004; Liaw, Huang e } \\
\text { Chen, 2007) }\end{array}$} & $\begin{array}{l}\text { Eu poderia ter aprendido a trabalhar com a EaD sozinho, utilizando } \\
\text { apenas manuais. }\end{array}$ & 0,814 & \multirow{2}{*}{0,500} \\
\hline & $\begin{array}{l}\text { Eu teria me sentido mais a vontade em trabalhar com EaD se alguém } \\
\text { tivesse me ensinado antes como proceder. }\end{array}$ & 0,814 & \\
\hline \multirow{3}{*}{$\begin{array}{c}\text { FACILIDADE DE USO } \\
\text { (Gong, Xu e Yu,2004; } \\
\text { Davis,1989; Venkatesh e } \\
\text { Morris, 2000) }\end{array}$} & Não tive qualquer dificuldade em aprender a utilizar a EaD. & 0,893 & \multirow{3}{*}{0,508} \\
\hline & Para mim, interagir com os alunos utilizando EaD é uma tarefa fácil. & $-0,381$ & \\
\hline & No geral, acho muito fácil trabalhar com a EaD. & 0,92 & \\
\hline \multirow{6}{*}{$\begin{array}{c}\text { UTILIDADE DE USO } \\
\text { (America,2006; Davis, } \\
\text { 1989) }\end{array}$} & Trabalhar com EaD me permitiu realizar tarefas mais rapidamente. & 0,796 & \multirow{6}{*}{0,686} \\
\hline & $\begin{array}{l}\text { Trabalhar com EaD aumentou a eficácia do meu trabalho como } \\
\text { professor. }\end{array}$ & 0,87 & \\
\hline & Trabalhar com EaD melhorou meu desempenho como professor. & 0,65 & \\
\hline & Trabalhar com EaD aumentou minha produtividade como professor. & 0,806 & \\
\hline & $\begin{array}{l}\text { Acredito que o método de ensino pela Internet é mais eficaz que a } \\
\text { modalidade presencial. }\end{array}$ & 0,459 & \\
\hline & $\begin{array}{l}\text { Eu acredito que interagir com os alunos utilizando a internet facilita } \\
\text { meu trabalho como professor. }\end{array}$ & 0,717 & \\
\hline \multirow{2}{*}{$\begin{array}{c}\text { INTENÇÃO DE USO } \\
\text { (America, 2006; Smarkola } \\
\text { 2008) }\end{array}$} & $\begin{array}{l}\text { Se for uma decisão que dependesse só de mim, eu trabalharia } \\
\text { novamente com a EaD. }\end{array}$ & 0,925 & \multirow{2}{*}{0,500} \\
\hline & $\begin{array}{l}\text { Eu pretendo continuar inserindo recursos de EaD em minhas } \\
\text { disciplinas tanto quanto possível. }\end{array}$ & 0,925 & \\
\hline
\end{tabular}

Fonte: Dados da Pesquisa

O Quadro 3 apresenta os coeficientes de consistência interna dos fatores, medidos por meio do teste Alpha de Cronbach. Adotando-se o ponto de consistência determinado por Hair et al. (2005) de o,6 para pesquisas exploratórias, pode-se observar que apenas os fatores originais do modelo TAM apresentam níveis satisfatórios de 
consistência. Por se tratar de uma pesquisa exploratória, sem pretensões determinísticas, optou-se por manter os fatores “Tempo" e “Autoeficácia” nos testes de hipóteses. Uma possível explicação para os valores abaixo do nível ideal indicado por Hair et al. (2005) pode ter ocorrido em decorrência do tamanho da amostra, que mesmo apresentando uma margem de erro significativamente reduzida para a população em estudo, ainda pode ser caracterizada como pequena para técnicas de análise multivariada.

Quadro o3-Consistência Interna dos Fatores

\begin{tabular}{|l|c|}
\hline \multicolumn{1}{|c|}{ Fator } & Alpha de Cronbach \\
\hline Tempo & 0,570 \\
\hline Autoeficácia & 0,489 \\
\hline Facilidade de Uso & 0,642 \\
\hline Utilidade de Uso & 0,809 \\
\hline Intenção de Uso & 0,829 \\
\hline
\end{tabular}

Fonte: Dados da Pesquisa

A análise de correlação de Pearson serviu como um passo inicial antes de ser realizada a regressão linear. Neste caso, a inexistência de correlações estatisticamente significantes entre as variáveis que compõem o conjunto de hipóteses do estudo já indicam que o modelo de regressão não será relevante para estas variáveis.

Seguindo essa linha de raciocínio, verificou-se que a Hi não pode ser aceita (Quadro 4, em amarelo), indicando, assim, que a restrição temporal não apresenta dependência linear significativa em relação a facilidade de uso do sistema. Esse resultado corrobora outros estudos, que também encontraram resultados semelhantes no que concerne à relação entre essas variáveis. No estudo de America (2006), a correlação entre tempo e facilidade de uso e utilidade de uso também não foi estatisticamente significante. 


\begin{tabular}{|c|c|c|c|c|c|}
\hline Fatores & Estatísticas & $\begin{array}{c}\text { Intenção } \\
\text { de Uso }\end{array}$ & Teтро & $\begin{array}{l}\text { Autoeficácia } \\
\text { Computacional }\end{array}$ & $\begin{array}{c}\text { Facilidade } \\
\text { de Uso }\end{array}$ \\
\hline \multirow{3}{*}{ Tempo } & $\begin{array}{l}\text { Pearson } \\
\text { Correlation }\end{array}$ & $0,558^{* *}$ & & & \\
\hline & Sig. (2-tailed) & 0 & & & \\
\hline & $\mathrm{N}$ & 76 & & & \\
\hline \multirow{3}{*}{$\begin{array}{l}\text { Facilidade de } \\
\text { Uso }\end{array}$} & $\begin{array}{l}\text { Pearson } \\
\text { Correlation }\end{array}$ & 0,119 & $-0,085$ & $0,550^{* *}$ & \\
\hline & Sig. (2-tailed) & 0,307 & 0,463 & 0 & \\
\hline & $\mathrm{N}$ & 76 & 76 & 76 & \\
\hline \multirow{3}{*}{ Utilidade de Uso } & $\begin{array}{l}\text { Pearson } \\
\text { Correlation }\end{array}$ & $0,547^{* *}$ & $0,395^{* *}$ & $0,360^{* *}$ & $0,343^{* *}$ \\
\hline & Sig. (2-tailed) & 0 & 0 & 0,001 & 0,002 \\
\hline & $\mathrm{N}$ & 76 & 76 & 76 & 76 \\
\hline
\end{tabular}

Fonte: Dados da Pesquisa.

As correlações apresentadas no Quadro 4 ainda revelam que o fator "Facilidade de Uso" não está correlacionado com a "Intenção de Uso" (em azul). Desta forma, $\mathrm{H}_{5}$ também não pôde ser verificada. Tal resultado caminha em sentido oposto ao postulado por Davis (I989).

A despeito da não confirmação de $\mathrm{H}_{1}$ e $\mathrm{H}_{5}$, as hipóteses $\mathrm{H}_{2}, \mathrm{H}_{3}, \mathrm{H}_{4}$ e $\mathrm{H} 6$ foram todas confirmadas por meio de regressões lineares simples (todos os coeficientes encontrados foram significativos), conforme apresentado no quadro 5. Desta forma, verificou-se a existência de relações de dependência linear positivas entre as variáveis analisadas e as variáveis dependentes utilizadas (utilidade de uso, facilidade de uso e intenção de uso). 
Quadro o5 - Regressões das Hipóteses Primárias

\begin{tabular}{|c|c|c|c|c|c|c|c|}
\hline \multicolumn{8}{|c|}{$\mathrm{H}_{2}$ - Variável Dependente: Utilidade de Uso } \\
\hline \multirow[t]{2}{*}{ Modelo } & \multicolumn{2}{|c|}{$\begin{array}{l}\text { Coeficientes Não } \\
\text { Padronizados }\end{array}$} & $\begin{array}{c}\text { Coeficientes } \\
\text { Padronizados }\end{array}$ & \multirow[t]{2}{*}{$\mathbf{t}$} & \multirow[t]{2}{*}{ Sig. } & $\mathbf{R}$ & $\mathbf{R}^{2}$ \\
\hline & B & Erro Padrão & Beta & & & \multirow{3}{*}{0,395} & \multirow{3}{*}{0,156} \\
\hline Constante & 0 & 0,106 & - & 0 & 1 & & \\
\hline Tempo & 0,395 & 0,107 & 0,395 & 3,7 & 0 & & \\
\hline \multicolumn{8}{|c|}{$\mathrm{H}_{3}$ - Variável Dependente: Facilidade de Uso } \\
\hline \multirow[t]{2}{*}{ Modelo } & \multicolumn{2}{|c|}{$\begin{array}{c}\text { Coeficientes Não } \\
\text { Padronizados }\end{array}$} & $\begin{array}{l}\text { Coeficientes } \\
\text { Padronizados }\end{array}$ & \multirow[t]{2}{*}{$\mathbf{t}$} & \multirow[t]{2}{*}{ Sig. } & $\mathbf{R}$ & $\mathbf{R}^{\mathbf{2}}$ \\
\hline & $\mathbf{B}$ & Erro Padrão & Beta & & & \multirow{3}{*}{0,550} & \multirow{3}{*}{0,303} \\
\hline Constante & 0 & 0,096 & - & 0 & 1 & & \\
\hline $\begin{array}{l}\text { Autoeficáci } \\
\text { a }\end{array}$ & 0,55 & 0,097 & 0,55 & 5,671 & 0 & & \\
\hline \multicolumn{8}{|c|}{$\mathrm{H}_{4}$ - Variável Dependente: Utilidade de Uso } \\
\hline \multirow[t]{2}{*}{ Modelo } & \multicolumn{2}{|c|}{$\begin{array}{l}\text { Coeficientes Não } \\
\text { Padronizados }\end{array}$} & $\begin{array}{c}\text { Coeficientes } \\
\text { Padronizados }\end{array}$ & \multirow[t]{2}{*}{$\mathbf{t}$} & \multirow[t]{2}{*}{ Sig. } & $\mathbf{R}$ & $\mathbf{R}^{2}$ \\
\hline & B & Erro Padrão & Beta & & & \multirow{3}{*}{0,360} & \multirow{3}{*}{0,130} \\
\hline Constante & 0 & 0,108 & - & 0 & 1 & & \\
\hline $\begin{array}{l}\text { Autoeficáci } \\
\text { a }\end{array}$ & 0,36 & 0,108 & 0,36 & 3,319 & 0,001 & & \\
\hline \multicolumn{8}{|c|}{ H $_{6}$ - Variável Dependente: Intenção de Uso } \\
\hline \multirow[t]{2}{*}{ Modelo } & \multicolumn{2}{|c|}{$\begin{array}{l}\text { Coeficientes Não } \\
\text { Padronizados }\end{array}$} & $\begin{array}{c}\text { Coeficientes } \\
\text { Padronizados }\end{array}$ & \multirow[t]{2}{*}{$\mathbf{t}$} & \multirow[t]{2}{*}{ Sig. } & $\mathbf{R}$ & $\mathbf{R}^{2}$ \\
\hline & B & Erro Padrão & Beta & & & \multirow{3}{*}{0,547} & \multirow{3}{*}{0,299} \\
\hline Constante & 0 & 0,97 & - & 0 & 1 & & \\
\hline $\begin{array}{l}\text { Utilidade de } \\
\text { Uso }\end{array}$ & 0,547 & 0,97 & 0,547 & 5,622 & 0 & & \\
\hline
\end{tabular}

Fonte: Dados da Pesquisa

Em relação à $\mathrm{H}_{2}$, obteve-se um resultado que evidencia a preocupação dos professores em torno de terem um tempo adequado para lidar com a tecnologia para ensinar a distância, ou seja, há a percepção de que ter tempo disponível é um fator importante para a percepção de utilidade de uso de um sistema, neste caso, como plataforma de ensino a distância, mais especificamente o Moodle.

Em relação às hipóteses $\mathrm{H}_{3}$ e $\mathrm{H}_{4}$, os resultados caminham em sentido semelhante aos achados de Gong et al. (2004) e Pituche Lee (2006) que constataram que a facilidade de uso percebida e a intenção comportamental de uso para o e-learning são influenciadas diretamente pela autoeficácia computacional, e que esta também influencia a intenção de uso do sistema. Desta forma, a percepção de autoeficácia 
computacional reflete diretamente as percepções de utilidade e facilidade de uso do sistema por parte dos docentes.

Por fim, H6, que trata da influência da utilidade de uso sob a intenção de uso do sistema, corroboram os resultados obtidos por Smarkola (2008), que utilizou o DTPB (Decomposed Theory of Planned Behavior), adaptado a partir da Teoria do Comportamento Planejado (TPB), e o do TAM, para investigar o comportamento e a intenção de uso da tecnologia por parte dos professores. Assim, ao perceberem que há uma utilidade de uso da tecnologia para ensinar a distância, os professores estariam dispostos a continuar a usar o sistema voluntariamente, mesmo sem ser uma obrigação mandatória, como no caso deste estudo, estando em linha com resultados anteriores de que a intenção de uso é positivamente influenciada pela percepção de utilidade do sistema.

\section{Considerações Finais}

Esta pesquisa investigou, a partir do modelo de aceitação da tecnologia (TAM), os fatores que influenciam a intenção dos professores participantes dos cursos a distância de uma universidade federal em continuar o uso do e-learning. A maior contribuição desta pesquisa foi verificar a pouca experiência dos docentes com o uso de tecnologia aplicada ao ensino e em lecionar na modalidade a distância, bem como o pouco tempo que trabalham na instituição e a utilização concentrada de apenas algumas das ferramentas disponibilizadas pelo ambiente virtual de aprendizagem Moodle. Ao avaliaras variáveis independentes autoeficácia computacional e tempo foi possível elucidar algumas questões importantes sobre a percepção de utilização de sistemas de informação no ambiente de ensino dentro do contexto brasileiro.

Em relação aos construtos comportamentais de utilização de sistemas de informação, verificou-se, por meio de uma série de indicadores, que os professores demonstram grande interesse em continuar lecionando no modelo de EAD, percebendo isso como útil para o exercício da docência. Esses indicadores ainda revelaram uma preocupação em relação à administração do tempo. O tempo é visto como algo que exige maior planejamento por parte dos docentes. 
No que concerne aos testes de hipóteses que nortearam a pesquisa, duas hipóteses não puderam ser aceitas, a de que a percepção de tempo para aprender a usar o sistema influencia a facilidade de uso do sistema e que a facilidade influencia a intenção de uso. Esta última constatação vai em direção oposta aos postulados teóricos que envolvem o modelo de aceitação de tecnologia utilizado como base no estudo. Isto pode indicar que, para professores que usam a tecnologia para a modalidade a distância, a facilidade de uso pode não ser um fator relevante para explicar a intenção em continuar o uso de plataformas de ensino a distância.

Devido à natureza exploratória descritiva da presente pesquisa, aliado às suas limitações, não é prudente abordar implicações prescritivas. Todavia, o presente estudo pode servir como uma guia para os gestores verificarem fatores que podem ser trabalhados em políticas de treinamento e capacitação dos professores. Em vista dos resultados obtidos, é possível sugerir mudanças quanto ao treinamento dos docentes na utilização das ferramentas do ambiente virtual. É necessário que os professores interajam com os alunos por meios de ferramentas que proporcionem um maior contato e, dessa forma sejam diminuídas as dificuldades existentes nessa modalidade de ensino.

A facilidade de utilizar o sistema mostrou-se ser uma variável importante para os professores, dessa forma, é importante que os docentes tenham contato com o sistema antes de trabalhar com o mesmo, para assim, identificar quais são as dificuldades e tentar amenizá-las antes de trabalhar online com os alunos.

\section{Limitações e Pesquisas Futuras}

A principal limitação desta pesquisa recai no fato de a mesma ser um estudo transversal simples que ocorreu por meio de uma amostragem não-probabilística, impossibilitando inferências, além do fato de abordar apenas professores que lecionavam no momento da realização da pesquisa. Em decorrência dos procedimentos amostrais utilizados, os resultados aqui encontrados não podem ser generalizados, mas podem levantar questionamentos que podem servir como orientação para a realização de pesquisas futuras.

Com o intuito de ampliar as discussões sobre o tema abordado nesta pesquisa e prosseguir com futuras abordagens seria interessante realizar outras pesquisas com 
amostras maiores fazendo com que o escopo das conclusões seja ampliado. Estudos que associem outros fatores/variáveis podem ser desenvolvidos, dessa forma novos resultados serão observados, assim como estudos que investiguem se há diferenças de comportamento na utilização de sistemas virtuais de aprendizagem entre professores que ministram disciplinas de naturezas diferentes.

\section{Referências}

AGARWAL, R.; PRASAD, J. Are individual differences germane to the acceptance of new information technologies? Decision Sciences, v. 3O, n. 2, p. 36I-9I, I999.

AGARWAL, R.; PRASAD, J. A field study of the adoption of software process innovations by information system professionals. IEEE Transactions on Engineering Management, v. 47 n. 3, p. $295^{-}$ 308,2000 .

AJZEN, I.The Theory of Planned Behavior.Organizational Behavior and Human Decision Processes, v. 5O, n. 2, pp. I79-2II, I99I.

AMERICA, C. Management Education Via The Internet: Factors Facilitating And Inhibiting The Adoption Of Webct At A Faculty In A Higher Education Institution. 2006. I2ff. Thesis Dissertation. University Of The Western Cape, Bellville, 2006.

ARBAUGH, J. B. Is There an Optimal Design for On-Line MBA Courses? Academy of Management Learning and Education, v. 4, n. 2, p. I35-I49, 2005.

BANDURA, A. Self-efficacy: toward a unifying theory of behavioral change. Psychological Review, v. 84, p: I9I-2I5, I977.

BANDURA, A. Social foundations of thought and action: a social cognitive theory.EnglewoodCliffs, NJ: Prentice Hall, s986.

BRAUER, M. Resistência à educação a distância na educação corporativa. Tese de Doutorado, Fundação Getulio Vargas, Departamento de Administração, 2008.

CHENG, B.; WANG, M.; YANG, S.; PENG, K. Acceptance of competency-based workplace elearning systems: Effects of individual and peer learning support. Computers \& Education, v. 57, n. 2, p. I3I7-I333, 2 OII.

CHILDERS, T.; CARR, C.; PECK, J.; CARSON, S. Hedonic and utilitarian motivations for online retail shopping behavior. Journal of Retailing, v. 77, n. 4, p. 51-35, $2 \mathrm{OII}$.

COMPEAU, D. R.; HIGGINS, C. A. Computer self-efficacy: development of a measure and initial test. MIS Quarterly, v. I9, n. 2, p. I89-2II, I995.

DAVIS, F. D. Perceived of use, and user acceptance of information technology.MIS Quartelly, Minneapolis, USA, v.I 3, n. 3, p. 319-338, r989.

DAVIS, F. D; BAGOZZI, R.; WARSHAW, P.R. User acceptance of computer technology: a comparison of two theoretical models. Management Science, v. 5, n.8, p. 982-IOO3, I989. 
DAVIS, F. D.; BAGOZZI, R. P.; WARSHAW, P. R. Extrinsic and intrinsic motivation to use computers in the workplace. Journal of Applied Social Psychology, v. 22, n. I4, p. IIII-II32, I992. FILATRO, A. Design instrucional contextualizado: educação e tecnologia. São Paulo: SENAC, 2003. FISHBEIN, M.; AJZEN, I. Belief, Attitude. Intention and Behavior: An Introduction to Theory and Research. Reading, MA: Addison-WesleyPublishing, I975.

FREITAS, A. 2009. A implementação do e-learning nas escolas de gestão: um modelo integrado para o processo de alinhamento ambiental. Rio de Janeiro, RJ. Tese de Doutorado em Administração de Empresas. PUC-Rio. 330 p.

GILBERT, S.; JONES, M. G. E-learning is e-normous. Electric Perspectives, v. 26, n.3, p. 66-82, 2OOI.

GONG, M,; XU, Y,; YU, Y. An enhanced technology acceptance model for web-based learning. Journal of Information Systems Education. v. I5, n. 4, p.365, 2004.

GURI-ROSENBLIT, S. Distance education and e-learning: not the same thing. Higher Education, v. 49, n. 4, p. 467-493, 2005.

HAIR, J.; ANDERSON, R.; TATHAN, R; BLACK, W. Análise Multivariada de Dados. 5 ed. Porto Alegre: Bookman, 2005.

HARASIM, L. Shift happens online education as a new paradigm in learning. Internet and Higher Education, v. 3, n.I-2, p: 4I-6I, 2000

HARASIM, L.; TELES, L.; TUROFF, M.; HILTZ, S. Redes de aprendizagem - Um guia para ensino e aprendizagem on-line, Ed. Senac: São Paulo, 2005.

HENRY, P. 2OOI. E-learning technology, content and services. Education + Training. v. 43, n.4, p.249-255.

HONG, J.; HWANG, M.; HSU, H.; WONG, W.; CHEN, M. Applying the technology acceptance model in a study of the factors affecting usage of the Taiwan digital archives system. Computers \& Education, v. 57, n. 3, p. 2086-2094, 2OII.

HUANG, A.; Yang, S.;Liaw, S.A study of user’s acceptance on situacionalmashups in situational language teaching.British Journal of Education Technology, v. 43, n.I, p.52, 2012.

IGBARIA, M.; GUIMARAES, T.; DAVIS, G. Testing the determinants of microcomputer usage via a structural equation model. MIS Quarterly, v. II, n. 4, p. 87-II4, I995.

JACKSON, C.; CHOW, S.; ROBERT, A. Towards an understanding of the behavioural intention to use an IS. Decision Sciences, v. 28, n. 2, p. 357-89, I997.

KIRAZ, E.; OZDEMIR, D.The Relationship between Educational Ideologies and Technology Acceptance in Pre-service Teachers. Educational Technology \& Society, v. 9, n. 2, p. I52-I65, 2006.

KULVIWAT, S.; BRUNER II, G. C.; KUMAR, A.; SUZANNE, A. N.; CLARK, T. Toward a Unified Theory of Consumer Acceptance Technology. Psychology and Marketing, v. 24, n. 12, p. I059-I084, 2007 .

MALHOTRA, N. Pesquisa de marketing: uma orientação aplicada. 4. ed. Porto Alegre: Bookman, 2006. 
MARTINS, L.L.; KELLERMANNS, F.W.A Model of Business School Students' Acceptance of a Web-Based Course Management System. Academy of Management Learning and Education, v. 3, n. I, p. 7-26, 2004.

MATHIESON, K. Predicting User Intention: Comparing the Technology Acceptance Model with the Theory of Planned Behavior. Information System Research, v. 2, p I73-I9I, I99I.

MOODLE. Moodle Docs. Disponível em: <http://docs.moodle.org/en/About_Moodle>. Acesso em: I8 Nov. 2009.

MOORE, G. C.; BENBASAT, I. Development of an instrument to measure the perceptions of adopting an information technology innovation. Information Systems Research, v.2, n. 3, p. 192-222, I99I.

NICHOLS, M. 2008. E-Primer Series - E-learning in context. Laidlaw College, New Zealand. Disponívelem: http://akoaotearoa.ac.nz/project/eprimerseries/resources/files/e-learningcontext-I-eprimer-series. Acesso em 03.09.2OII

PITUCH, K. A.; LEE, Y.The influence of system characteristics on e-learning use. Computer \& Education, v. 47, n. 2, p. 222-244, 2006.

RAMOS, A. S. M.; OLIVEIRA, B. M. K. Diferenças de Gênero na Aceitação de um Ambiente Virtual de Aprendizado: um estudo comgraduandos do curso de Administração na modalidade a distância. Revista Ibérica de Sistemas e Tecnologias de Informação, n. ${ }^{\circ}$ 5, 6/20IO. Disponível em: $<$ http://dialnet.unirioja.es/servlet/articulo? codigo $=3700986>$ Acesso em: IO/IO/2OI2.

ROGERS, E. Diffusion of Innovations. 5 ed. New York: Free Press, 2003.

ROSENBERG, M. Além do e-learning: abordagens e tecnologias para a melhoria do conhecimento e do desempenho organizacional. Rio de Janeiro: Qualitymark, 2008.

SANCHEZ-FRANCO, M.J. WebCT - The quasimoderating effect of perceived affective quality on an extending Technology Acceptance Model. Computers \& Education, v.54, n.I, p: 7-46, 2 OIO.

SIRITONGTHAWORN, S.; KRAIRIT, D.; DIMMITT, N. J.; PAUL, H. The study of e-learning technology implementation: A preliminary investigation of universities in Thailand. Education and Information Technologies, v. II, n. 2 p. I37-I6o.

SMARKOLA, C. Efficacy of a planned behavior model: Beliefs that contribute to computer usage intentions of student teachers and experienced teachers. Computers un Human Behavior, v. 24, p. II96-I2I5. 2008.

SUGAR, W.; CRAWLEY, F.; FINE, B. Critiquing Theory of Planned Behaviour as a method to assess teachers' technology integration attitudes. British Journal of Educational Technology, v.36, n.2, p. 33I-334, 2005 .

SUH, B.; HAN, I.The impact of customer trust and perception of security control on the acceptance of electronic commerce. International Journal of Electronic Commerce, v.7, n.3, p. I35-6I, 2003.

TAYLOR, S.; TODD, P. A. Assessing IT Usage: the role of prior experience. MIS Quarterly, v. I9, n. 2, p. 56I-57O, I995a.

TAYLOR, S.; TODD, P. A. Understanding Information Technology Usage: A Test of Competing Models. Information Systems Research, v. 6, n. 4, p. 144-176. 1995 b. 
TEO, T.; NOYES, J. An assessment of the influence of perceived enjoyment and attitude on the intention to use technology among pre-service teachers: A structural equation modeling approach. Computers \& Education, v. 57, n. 2, p. I645-I653, 2 OII.

THOMPSON, R.; HIGGINS, C.; HOWELL, J. Personal computing: toward a conceptual model of utilization. MIS Quarterly, v. I5, n. I, p. I25-I43, I99I.

THOMPSON, R.; HIGGINS, C.;HOWELL, J. Influence of Experience on Personal Computer Utilization: Testing a Conceptual Model. Journal of Management Information Systems, v. II, n. I, p. I67-I87, I994.

TRIANDIS, H. C. Interpersonal behavior. Monterey, Canada: Brooke/Cole, I977.

VASCONCELLOS, L. Adoção de inovações: o uso do e-learning por colaboradores de uma empresa de telecomunicações. Tese de Doutorado, Universidade de São Paulo, Departamento de Administração, 2008.

VENKATESH, V. Creation of favorable user perceptions: exploring the role of intrinsic motivation. MIS Quarterly, v. 23, n. 2, p. 239-6o, I999.

VENKATESH, V.; DAVIS, F. D. A theoretical extension of the technology acceptance model: Four longitudinal field studies. Management Science, v. 46, p. I86-204, 2000.

VENKATESH, V.;MORRIS, M.; DAVIS, G.; DAVIS, F. User Acceptance of Information Technology: Toward a Unified View. MIS Quarterly, v.27, n.3, p.425-478, 2003.

YOUSAFZAI, S.; FOXALL, G.; PALLISTER, J. Technology Acceptance: a Meta-Analysis of the TAM: Part I. Journal of Modeling in Management, v.2, n.3, p. 25 $\mathrm{I}^{-280}, 2007$.

ZEMSKY, R.; MASSY, W. 2004.Thwarted Innovation: What happened to e-learning and why. A Final Report for The Weatherstation Project of The Learning Alliance at the University of Pennsylvania in cooperation with the Thomson Corporation. The Learning Alliance for Higher Education. PennsylvaniaUniversity. 